\title{
ÜBER DIE EIGENART DER AFRIKANISCHEN PYGMAEN*
}

\author{
Von Martin Gusinde
}

Mit 3 Abbildungen

Im gestaltenreichen und formengebildlichen Vielerlei der menschlichen Rassen und Volksgruppen stellen die Pygmäen in der afrikanischen Tropenzone eine, wie man sagen möchte, vom durchgängigen Grundriß weit abweichende Körpermodelung zur Schau. Mag auch ihr deutlich ungewöhnliches Äußeres, eben weil unübersehbar augenfällig, von den Beobachtern am häufigsten besprochen und am liebsten erörtert worden sein; als befremdlich im gleichen Ausmaß spricht ihre Daseinsweise an, die man richtig als Wechselwirkung zu ihrer nahezu menschenfeindlichen Umwelt deuten muß.

Nur mit echten Pygmäen, den blutreinen Rassezwergen, haben wir es hier zu tun, weswegen weder die pathologische Verkrüppelung einzelner Personen mit chondrodysthrophischem oder mit hypophysär-diencephalem Zwergwuchs - dem auch der sogenannte heredo-degenerative angegliedert wird(1)** — noch die allein durch verminderte Körperhöhe gekennzeichnete Konstitutionsanomalie des sogenannten primordialen Zwergwuchses zur Erwähnung gelangt(2). Sicheres Wissen um das Vorhandensein echter Pygmäen besaßen bereits die alten Ägypter, die das "Gebiet der Nilquellen» als deren Wohnraum ausgegeben haben. Ebenso überrascht der geschichtliche Nachweis, daß sie mit bestimmter Deutlichkeit diese erbgenetisch festgefügte Zwergform von der pathologischen Deformation vereinzelter Krüppel unterschieden haben(3).

Neuzeitlichen Forschungen ist es endlich gelungen, den Wohnbereich der echten Pygmäen genau abzustecken und ihn selbst in seiner natürlichen Eigenart ausführlich zu bestimmen. Nun steht fest, daß diese sonderbar geformten Zwergmenschen als richtige Waldbewohner im tropischen Bereich beheimatet sind. Weil es bekanntlich zuweilen schwerfällt, die sich nahe oder näher stehenden Formenkreise, Rassen und Subrassen gegeneinander abzugrenzen, braucht es nicht zu verwundern, daß jemand ein bestimmtes Volk den echten Pygmäen zurechnet, das indes von einem andern Beurteiler als pygmoide oder kurzweg als negride Varietät veranschlagt wird. Selbstverständlich scheiden bei diesen Erörterungen die Erscheinungen echter Variationsbildung aus, wie sich solche in der Südsee nachweisen lassen(4); vieles spricht dafür, $\mathrm{da} ß$ sämtliche dort wohnhaften Kleinwuchsformen tatsächlich nichts anderes darstellen als ortsbedingte phänotypische Anpassungen. Die Aëta auf den Philippinen, vielleicht besser bekannt unter der Benennung «Negritos», beurteile ich schon seit Jahr und Tag ebensowenig wie die Semang und Senoi auf Malakka als echte Pygmäen; wem es so beliebt, der mag sie in die Pygmoiden oder in einen anderen rassischen Formerikreis einreihen. Wegen mehrerer entscheidender Körpermerkmale, auch wegen der Grundstruktur der Wirtschaftsform im heimatlichen Urwald, kommen die Andamanesen den zentralafrikanischen Pygmäen sehr nahe, während von diesen letzteren die in eine gänzlich andersgeartete Umwelt hineingestellten Buschmänner und Hottentotten Südafrikas mit bedeutsamen Sonderbildungen weit abweichen. Mithin sind es nahezu ausschließlich die bekannten Kleinwuchsgruppen im afrikanischen Tropenwalde, die eine eindeutige pygmäische Körperprägung zeigen.

* Die Abhandlung ist die Zusammenfassung eines Vortrages vor der Geographisch-Ethnographischen Gesellschaft Zürich am 18. März 1949.

** Die Ziffern in Klammern beziehen sich auf das Literaturverzeichnis am Schluß des Artikels. 
Nachdem hiermit sehr allgemein der Wohnbereich der echten Pygmäen geographisch abgegrenzt worden ist, sei nochmals darauf hingewiesen, daß der tropische Urwald ihren Lebensraum ausmacht. Dieser Blätterwald ist ein floristisches Gebilde eigentümlichster Zusammensetzung. Gewaltige Baumriesen, deren dicke Stämme eng nebeneinanderstehen und gleich mächtigen Säulen durchwegs kerzengrade nach oben streben, vereinigen sich in vielen Millionen zur immergrünen zentralafrikanischen Hyläa. Sie erstreckt sich nahezu ununterbrochen vom Großen Graben im Osten durch den breiten Kontinent noch weit über Kamerun hinaus nach Westen. In einer Höhenlage von etwa $40 \mathrm{~m}$ über dem Erdboden strecken sich weit die ausgreifenden Äste in beträchtlicher Breite aus und verflechten sich miteinander zu einem eng verknäulten, tief geschichteten Dach, in das sich, zu noch engerer Füllung und kompakter Verdichtung, Parasiten und Epiphyten, Schmarotzer und Schlingpflanzen aller Arten überwuchernd einlassen. Mit alledem wird für die in der Äquatorzone lichtstarken Sonnenstrahlen ein Durchdringen der von den Baumstämmen hoch oben gestützten flachdachartigen Baumkronenschicht in solch erheblichem Ausmaß unterbunden bzw. behindert, daß als Folge dessen darunter, im eigentlichen Waldraume, nur ein mattes, graues Schummerlicht aufkommt. Die dort heimischen zwerghaften Waldinsassen erfahren ihr ganzes Leben lang nicht mehr als eine bloß kurzfristige Belichtung mit sehr schwacher Strahleneinwirkung auf die Körperhaut. Im schmalen Landstreifen zu beiden Seiten des Äquators geben sich keine jahreszeitlichen Unterschiede atmosphärischer Art spürbar zu erkennen; dort gleicht ein Tag dem andern in allem und jedem. Die Temperatur bewegt sich - von gelegentlichen Schwankungen bis höchstens fünf Grad abgesehen alltäglich zwischen dem 20. und 32. Grade als Minimum und Maximum. Damit geht ein buchstäblich alltäglicher Regen einher, den die schnell aufsteigenden Wolkenballen durchwegs in überreichlichen Wassermassen aus. schütten. $\mathrm{Ob}$ er nun den ganzen Tag oder bloß für wenige Stunden rauschend niederprasselt, er überschwemmt jedesmal den Erdboden, daß sich dieser oftmals und strichweise rasch ineinen richtigen

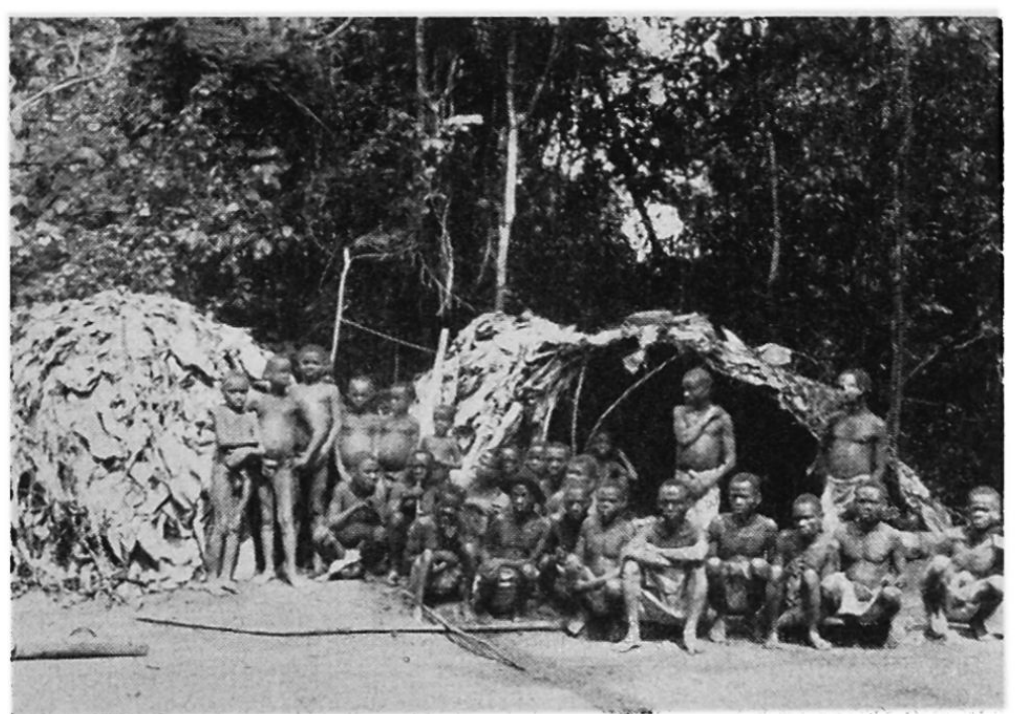

Abb. 1. Bambuti-Pygmäen vor ihren Hütten. Photo: M. Gusindf, Wien Sumpf umwandelt. Da, einer Zelt-

leinwand vergleichbar und von den gewaltigen Baumsäulen getragen, hoch oben das dichte Blätterdach der eng miteinander verknäulten Baumkronen sich hinzieht, kommt kein kräftiger Durchzug der Luft zustande; im ganzen wird die Verdunstung fast völlig behindert, und eine unvorstellbare Feuchtigkeit bleibt als Dauerzustand zurück. Demzufolge tropft und trieft es unaufhörlich; alle Gerätschaften und Gebrauchsgüter der Eingeborenen verfaulen oder vermodern, werden rostig und schimmelig. Sehr schwache Belichtung, gesteigerte Temperatur und ein Höchstmaß von Luftfeuchtigkeit sind bestimmende Eigentümlichkeiten des Lebensraumes, in dem die echten Rassezwerge ihr Dasein verbringen(5).

In der weitgespannten zentralafrikanischen Hyläa trifft der Europäer zu seiner eigenen Überraschung heutigentags echte Neger verschiedener Körperprägung als Nachbarn der Pygmäen an. Dieses Nebeneinander reicht verschiedenzeitlich tief, jedoch kaum irgendwo mehr als 300 Jahre für die ersten neuen Eindringlinge zurück. Als solche müssen alle gegenwärtig im Urwalde siedelnden Neger gelten; denn ursprünglich war die offene Steppe ihre Heimat, in der die wesentlichen Merkmale ihrer negriden Körperform gezüchtet worden sind(6). In den meisten Waldbezirken sind diese neuen Ansiedler erst seit wenigen Generationen seßhaft. Unwidersprochen eindeutig wird auf dieser und jener Seite die Tatsache bestätigt, daß die Pygmäen allein als erste und uralte Inhaber des Waldesraumes ansässig waren, weswegen man ihren kriegerischen Widerstand gegen die negerischen Eindringlinge als durchaus gerechtfertigt beurteilt(7). Unsere zwerghaften Waldmenschen, weniger wirksam ausgerüstet als ihre Gegner, unterlagen in diesem ungleichen Ringen; danach hat sich langsam ein friedfertiges Nebeneinander beider Volksgruppen ausgebildet, und man kann es als symbiotische Interessengemeinschaft veranschlagen, insofern der eine Teil vom andern zumindest einigermaßen vorteilhaft bedient wird. Damit hat die völkische und rassische Selbständigkeit beider Abteilungen keinesfalls eine ihre ursprüngliche Eigenart auflösende Einbuße erlitten. Allerdings wickeln sich da und dort seit zwei oder drei Generationen eigentliche Zwischenheiraten in der Weise ab, daß Pygmäenmädchen von Negern geehelicht werden; diese Mütter verbleiben samt ihren Kindern in den Dörfern der letzteren(8). 
Die wirtschaftliche Selbständigkeit, bei der die jetzt im Urwalde wohnhaften Neger verblieben sind, braucht hier nicht ausführlich geschildert zu werden; sie haben die kennzeichnende Grundform, den Hackbau, in die neuen Umweltbedingungen ertragsreich eingepaßt.

Das Wirtschaften der Pygmäen ist das freibeuterische Sammeln und Jagen, mit dem sich folgerichtig eine ausschließlich nomadisierende Lebensweise verbindet, im ganzen ihre eigene Schöpfung unter dem Zwange der übermächtigen, gewalttätigen Umwelt. Zwangsläufig verteilt sich die Gesamtheit der afrikanischen Pygmäen als mehrere selbständige Volksstämme über die breitgezogene tropische Hyläa, und ein jedes dieser Völker - fern davon, eine geschlossene Einheit zu bilden — steht da als eine beträchtliche Anzahl kleiner Horden, die ohne gegenseitige Verbindung lose nebeneinanderleben. Solch eine Horde gibt sich als Zusammenschluß einiger Familien $\mathrm{zu}$ erkennen, welch letztere sich gegenseitig verwandt fühlen und einander unter-

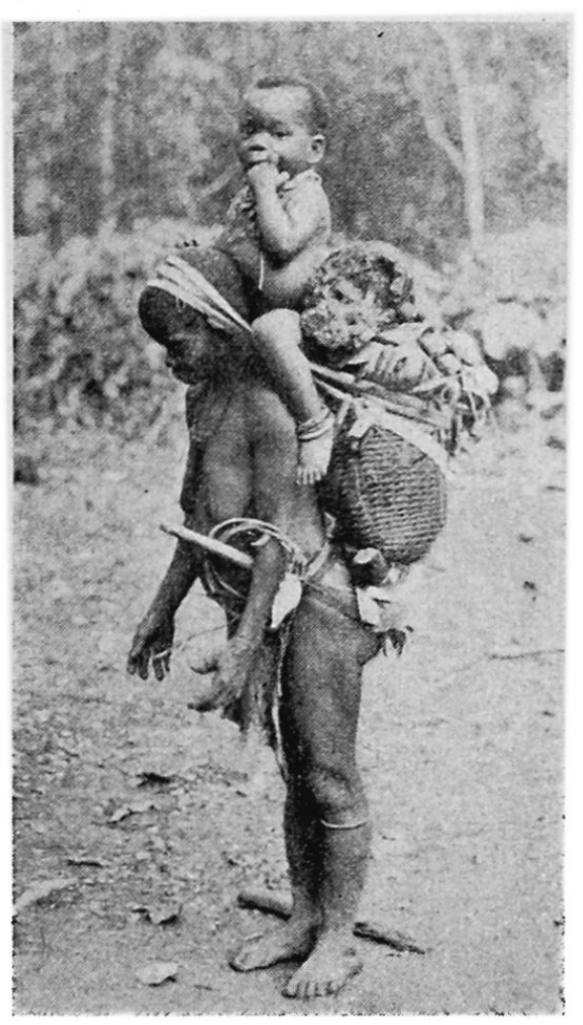

Abb.2. Bambuti-Pygmäin beim Sammeln. Photo: M. Gusinde, Wien stützen; einem besonderen Häuptling oder autoritativen Führer unterstehen sie nicht. Ebensowenig wird das gesamte Pygmäenvolk von einer alle Einzelhorden zusammenfassenden Autoritätsperson mit irgendwelcher Machtbefugnis geleitet oder regiert. Als vorzüglich begründete und festgefügte gesellschaftliche Vereinigung besteht allein die monogame Einzelehe, die, nach vorausgegangener völlig freier Gattenwahl, sich aus den beiden Eltern mit ihren Kindern zusammensetzt und im Volksganzen bzw. in der eigenen Horde unbehinderter Freizügigkeit und zwangloser Ungebundenheit erfreut. Dank dem unentbehrlichen Beitrag, den jede Frau zum Unterhalt ihrer Familie leistet, nimmt sie eine ihrem Gatten nahezu gleichrechtliche und durchaus ehrenvolle Stellung ein. Wie schon angedeutet wurde, besteht der pygmäische Wirtschaftsbetrieb im Sammeln und Jagen. Mithin leben unsere zwerghaften Waldmenschen buchstäblich von der Hand in den Mund; denn durchwegs ist der Ertrag ihres wirtschaftlichen Mühens dermaßen dürftig, daß an keinem Tage nach der Hauptmahlzeit ein beachtlicher Restbestand übrigbleibt, sogar sehr häufig die meisten Leute keine volle Sättigung erreichen. Im ganzen gesehen, machen die Pygmäen mehrmals im Jahre lange Zeiten einer ungenügenden Ernährung durch; dann und wann quält sie sogar empfindlicher Hunger. Mutter Natur benimmt sich im dortigen Urwalde sehr geizig und kargt unerbittlich mit den Nahrungsstoffen, die das Leben der zwerghaften Insassen erhalten. Viel zu wenig ist den Europäern bekannt, daß Tiere höherer Ordnungen bloß vereinzelt auftreten. Allein, die Zwergantilopen machen eine Ausnahme, und sie sind das alltägliche Ziel der Jäger. Daneben spüren diese auch den Schlangen und Eidechsen, den Wildkatzen und Klippschliefern nach; selten glückt es einem gewandten Weidmanne, den nicht ungefährlichen Elefanten zur Strecke zu bringen. Ihrerseits sammeln die Frauen Tag um Tag allerlei Kleingetier, vor allem Raupen und fette Larven, dicke Würmer und Ameisen, faustgroße Schnecken und plumpe Frösche, nebst Pilzen und Honig. Da sie das Töpfern nicht verstehen, kommen sie zu keinem eigentlichen Kochen. Ihre Lebensmittel bereiten sie nach dem höchst einfachen Verfahren zu, daß sie Fleischstücke zum Rösten auf glühende Holzkohle oder in heiße Asche legen, alles Kleingetier in harte grüne Blätter beutelartig einhüllen und nahe dem Feuer dünsten lassen. Als einziges Getränk steht ihnen natürliches Wasser zur Verfügung. 
Die unstete Lebensführung der niederen Sammelwirtschaft schließt unabdingbar feste Wohnstätten und auf Dauer berechnete Siedlungen aus. An jedweder geeigneten Stelle, je nachdem augenblickliche Gegebenheiten es an jedem Tage empfehlen, schlägt die Einzelfamilie, fast regelmäßig im Zusammenschluß mit ihrer Horde, eine einfache, vorwiegend kuppelförmige Laubhütte auf; darin verbringt sie eine oder zwei Nächte und zieht wieder weiter. Umweltbedingt verzichten die Pygmäen auf eigentliche Kleidung als Körperschutz und begnügen sich mit einem einfachen Schamschurz aus Bast; ihr Schmuckbedürfnis ist kaum nennenswert.

Wie ersichtlich, macht ihr Besitz an gegenständlichen Gütern das Mindeste aus, dessen ein Mensch zu seinem Bestehen bedarf. Der Mann verfügt bloß über Bogen und Pfeile, die in einem kurzen Köcher stecken; häufig trägt er auch einen Speer und einen Ledergürtel. Noch weniger Dinge nennt die Frau ihr Eigentum; denn es umfaßt allein einen großmaschigen Tragkorb, einen breiten Lederstreifen, der den auf ihrer linken Hüfte reitenden Säugling trägt, und manchmal einige sehr schlichte Zierstück-

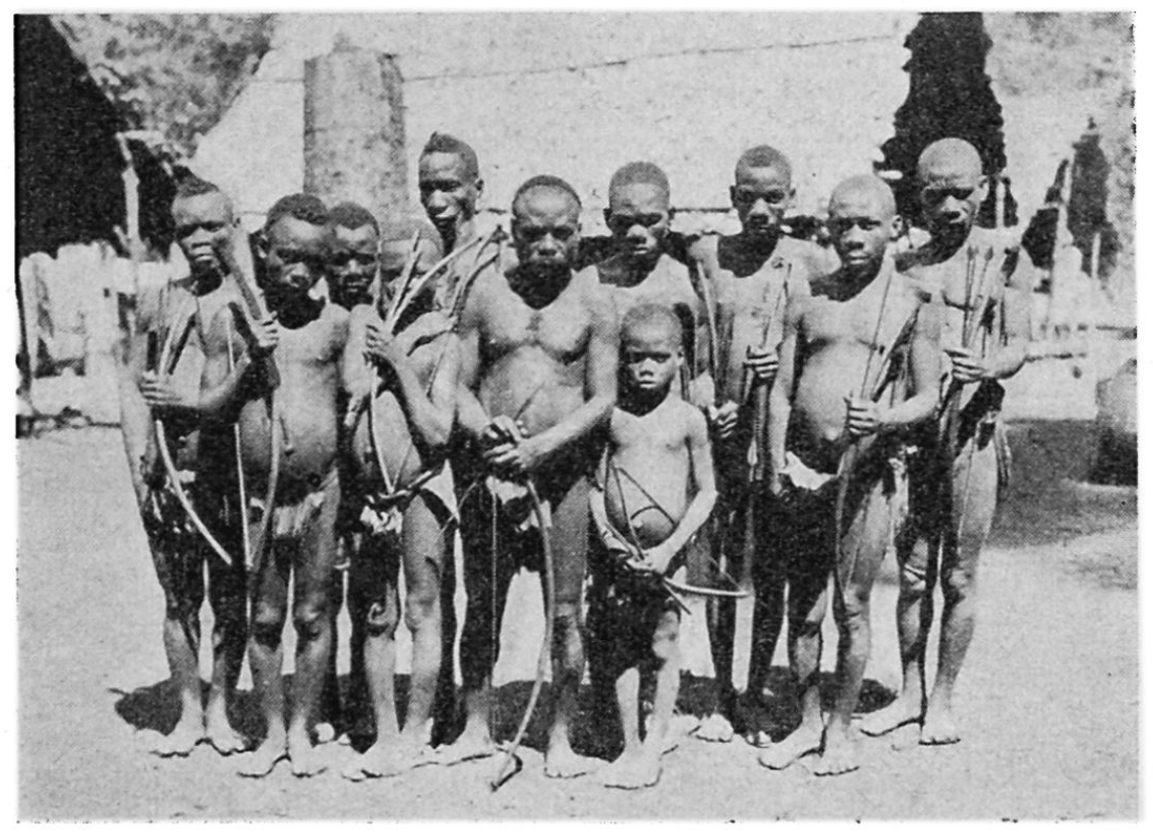

Abb.3. Gruppe von Bambuti-Pygmäen. Photo: M. Gusinde, Wien

chen. Denkbar vorteilhaft und mithin zu äußerster Dürftigkeit abfallend haben sich die Pygmäen in ihrer menschenfeindlichen Umwelt eingerichtet; die grundlegende Führung ihres gesamten Wirtschaftens betrachte ich als eine geniale Schöpfung im Sinne eines «Optimum adaptationis».

Am meisten auffällig wirken die afrikanischen Waldmenschen durch ihre gesamte Körperform. Mit ihrєm kennzeichnenden Zwergwuchs haben sie sich die Bewertung "Pygmäen» verdient; doch wäre es gänzlich verfehlt, darin allein ihre rassische Eigenart begründet zu sehen. Ihr zusammengefaßtes Äußeres und die aufeinander abgestimmten Merkmale geben als Formenganzheit den eigentlichen Pygmäentypus ab(9). Demnach käme es einem Mißbrauch der Bezeichnung «Pygmäen» gleich, letztere einer beliebigen Volksgruppe allein wegen der niedrigen Körperhöhe ihrer Mitglieder zuzuerkennen. Mit der unseren afrikanischen Waldmenschen eigentümlichen, innerhalb der vorgeschichtlichen und jetztzeitlichen Menschheit absolut geringsten Körperhöhe - als Durchschnittsmaß habe ich $143 \mathrm{~cm}$ für Männer und $137 \mathrm{~cm}$ für Frauen berechnet - verbinden sich arteigene Proportionsverhältnisse, und zwar erscheint der Kopf übermäßig dick, die zarten Arme sind zu lang und die zierlichen Beine zu kurz. Der niedrigen Gesamtgestalt und den leichten Knochen entspricht das sehr geringe Körpergewicht. Nicht minder überrascht die helle Färbung der Körperhaut, bei der es sich um ein bräunliches Gelb handelt; es sticht sehr deutlich vom tiefen Braun der benachbarten Neger ab.

In einzigartiger Sonderprägung zeigen sich die Abschnitte des Gesichtes. Die Stirn zieht gerade aufsteigend hoch hinauf und besitzt meistens eine stark konvexe Ausbeulung ihres gesamten Mittel- 
teiles. Über den unteren Stirnrand ziehen dicke Hautfalten unregelmäßig zur Nasenwurzel, die gänzlich abgeflacht liegt; daneben öffnen sich weit die Lider und lassen jeden Augapfel gleichsam quellend heraustreten. Als sonderbares Gebilde von einzigartiger Modelung sitzt eine unförmliche, beträchtlich in die Breite ziehende Nase im Mittelgesicht, das an sich, gegenüber dem hohen Ober- und Untergesicht, niedrig ist und wegen der mächtigen Nase noch niedriger anspricht. ImFormgefüge dieser letzteren geben sich zwei grundlegende Ausprägungen zu erkennen, die man als Knopfnase und als Trichternase umschreiben darf; rund um sie beide stellen sich noch vielerlei ähnliche Gestaltungen. Mit einer bloß mäßigen Prognathie verbindet sich das konvexe Vorwölben der Integumental-Oberlippe; sich angleichend an die helle Körperhaut, zeichnet sich in die durchwegs mitteldicken Schleimhautlippen ein frisches Rosa. Andere bedeutsame Eigenheiten hier übergehend, sei bloß noch die als einzigartig herausgestellte Blutgruppenformel erwähnt(10). Alles in allem: die afrikanischen Pygmäen sind durch arteigene Spezialisierung vieler Körpermerkmale gekennzeichnet; sie alle zur gestaltlichen Ganzheit zusammengefaßt, räumen diesem pygmäischen Formenkreise eine deutliche Sonderstellung im Vielerlei der menschlichen Rassen ein.

$\mathrm{Zu}$ diesen Wesenszügen der charakteristischen Pygmäenform hat sich bei den verschiedenen Lokalgruppen manche stammeseigene Prägung des einen und anderen Merkmals gesellt, und zwar vermutlich ortsbedingt. Beispielweise tritt im Westen eine etwas dunkle Hautfarbe und eine sehr wenig gesteigerte Körperhöhe gegenüber den östlichen Pygmäen auf. Dergleichen Abweichungen der einen Gruppe von der anderen als Ergebnis einer Bastardierung mit Negern auszugeben, erachte ich als ein leichtfertiges Unterfangen. Wo eine Blutmischung sich nicht sicher erweisen läßt, müssen die aufgezeigten Verschiedenheiten aus erbgenetischem Geschehen erklärt werden.

Wie ich es als wahrscheinliche Entwickung glaubhaft zu machen versuche, ist das mehrgestaltige Rassebild der afrikanischen sowie der außerafrikanischen Volksstämme mit pygmäischer Körperformung als Rassen oder Unterrassen, als Varietäten oder Typen, samt der ausgeglichen vorteilhaften Anpassung an die jeweilige Umwelt, das Wirkergebnis einiger selbständiger Isolate, welch letztere sich von der ehedem gar nicht differenzierten proto-negriden Schicht abgesondert haben, wobei in jedem einzelnen dieser Isolate echte Mutationen zur bestmöglichen Adaptation hin, unterstützt von ortsgebundenen Selektionsvorgängen, entscheidend mitgespielt haben(11).

Literatur: 1 HanharT, E.: Über heredo-generativen Zwergwuchs mit Dystrophia adiposogenitalis. Archiv der Julius-Klaus-Stiftung für Vererbungsforschung, I, 1925, S. 181-257. - 2 GüNTER, H.: Proportionsstudien an Zwergen. Virchows Archiv, 307, 1941, S. 641-653. - 3 GusINDE, M.: Die Kongo-Pygmäen in Geschichte und Gegenwart. Nova Acta Leopoldina, XI, 1942, S. 147-415. 4 Sperser, F.: Die Pygmäenfrage. Experientia, II, 1946, S. 1-16. - 5 Gusinde, M. (gemeinsam mit F. LAUSCHER): Meteorologische Beobachtungen im Kongo-Urwald. Sitzungsbericht der Akademie der Wissenschaften in Wien, Mathematisch-naturwissenschaftliche Klasse, Abt. IIa, Bd. 150, 1941, S. 281 bis 347. - 6 ReCHE, O.: Herkunft und Entstehung der Negerrassen. Beiträge zur Kolonialforschung, Tagungsband I, 1943, S. 152-168. - 7 MoelLER, A.: Les grandes lignes des migrations des Bantous de la province orientale du Congo belge. Bruxelles 1936. - 8 Gusinde, M.: Pygmäen-Neger-Bastarde im östlichen Kongogebiet. Zeitschrift für Morphologie und Anthropologie, XL, 1942, S. 92-148. 9 Gusinde, M.: Urwaldmenschen am Ituri. Anthropo-biologische Forschungsergebnisse bei Pygmäen und Negern im östlichen Belgisch-Kongo aus dem Jahre 1934/35. Wien 1948. - 10 JaDIN, J.: Les groupes sanguins des Pygmées. Mémoires de l'Institut Royal Colonial Belge. Bruxelles 1935. - GusINDE, M.: Erforschung der Bambuti-Pygmäen und ihrer Blutgruppen. Zeitschrift für Rassenphysiologie, VIII, 1936, S. 12-20. - 11 Gusinde, M.: Die menschlichen Zwergformen. Anthropo-biologische Erörterungen um die Pygmäen Afrikas. Experientia, V, 1949. - GusindE, M.: Die Twa-Pygmäen in Ruanda. Forschungsergebnisse im tropischen Afrika aus dem Jahre 1934. Wien-Mödling 1949.

\section{SUR LES CARACTÉRISTIQUES DES PYGMÉES AFRICAINS}

La composition réunit les résultats d'une enquête de campagne de longues années au sujet de pygmées africains. Ils permettent de voir que les habitants nettement nomadiques de la forêt de pluie tropicale sont des recueilleurs et chasseurs. Leur base sociale est le mariage resp. la famille monogame, dont existent de nombreux groupes isolés de caractère anthropologique local.

\section{SULLE CARATTERISTICHE DEI PIGMEI AFRICANI}

Lo scritto riassume i risultati di lunghi anni di osservazioni sui pigmei africani. Essi sono gli abitanti della foresta umida tropico-equatoriale, raccoglitori e cacciatori, nomadi per eccellenza, viventi in orde. L'organizzazione è caratterizzata dalla monogamia nel matrimonio; le famiglie si riuniscono in numerosi gruppi antropologici locali e isolati. 